\title{
Tipificaciones en Azorelloideae (Apiaceae) para la Flora de Argentina
}

\author{
Typifications in Azorelloideae (Apiaceae) for the Flora of \\ Argentina
}

\author{
Martina Fernández ${ }^{1 *}$ y Carolina I. Calviño ${ }^{1 *}$ (D)
}

\begin{abstract}
1. Instituto de Investigaciones en Biodiversidad y Medioambiente (INIBIOMA), Universidad Nacional del Comahue-CONICET, Quintral 1250, 8400 Bariloche, Río Negro, Argentina.
\end{abstract}

*mfernandez@comahue-conicet. gob.ar; ccalvino@comahueconicet.gob.ar

\section{Citar este artículo}

FERNÁNDEZ, M. \& C. I. CALVIÑO. 2020. Tipificaciones en Azorelloideae (Apiaceae) para la Flora de Argentina. Bol. Soc. Argent. Bot. 55: 131-136.

DOI: https://doi. org/10.31055/1851.2372.v55. n1.25026

\section{SUMMARY}

Background and aims: Azorelloideae is one of the four monophyletic subfamilies of Apiaceae and currently comprises 17 genera. In the context of the revision of the subfamily Azorelloideae (Apiaceae) for the Flora of Argentina project, 15 names belonging to Asteriscium, Bolax, Bowlesia, Diposis and Pozoa were found to need typifications.

M\&M: To resolve typifications, all protologues and the original material of all names were studied, and decisions were made following the International Code of Nomenclature for Algae, Fungi and Plants, and based on our expertise on the taxonomy of the group.

Results \& Conclusions: 14 lectotypes and one neotype are designated, and justifications are given for each selection. As a result, a permanent reference specimen has been selected for all names of Azorelloideae included in Flora Argentina, which is an essential step for the correct identification of species.

\section{KEY WORDS}

Asteriscium, Bolax, Bowlesia, Diposis, nomenclature, Pozoa, Umbelliferae.

\section{RESUMEN}

Introducción y objetivos: Azorelloideae es una de las cuatro subfamilias monofiléticas de Apiaceae y actualmente comprende 17 géneros. En el contexto de la revisión de la subfamilia para el proyecto de Flora Argentina, se encontraron 15 nombres pertenecientes a Asteriscium, Bolax, Bowlesia, Diposis y Pozoa, que requieren tipificaciones.

M\&M: Para resolver las tipificaciones, se examinaron los protólogos y el material original de todos los nombres, y las decisiones se tomaron siguiendo el Código Internacional de Nomenclatura para Algas, Hongos y Plantas, y a partir de nuestro conocimiento de la taxonomía del grupo.

Resultados y conclusiones: Se designan 14 lectotipos y un neotipo, y se brindan justificaciones para cada selección. Como resultado, todos los nombres de Azorelloideae incluidos en Flora Argentina, tienen seleccionado un ejemplar permanente de referencia, lo cual es un paso esencial para la correcta identificación de las especies.

Palabras clave

Asteriscium, Bolax, Bowlesia, Diposis, nomenclatura, Pozoa, Umbelliferae.

\section{INTRODUCCIÓN}

Azorelloideae es una de las cuatro subfamilias monofiléticas de Apiaceae y actualmente comprende 17 géneros (Nicolas \& Plunkett, 2009; Plunkett \& Nicolas, 2017): Asteriscium Cham. \& Schltdl., Azorella Lam., Bolax Comm. ex Juss., Bowlesia Ruiz \& Pav., Dichosciadium Domin, Dickinsia Franch., Diplaspis Hook. f., Diposis DC., Domeykoa Phil., Drusa DC., Eremocharis Phil., Gymnophyton Clos, Homalocarpus Hook. \& Arn., Klotzschia Cham., Oschatzia Walp., Pozoa Lag. y Spananthe Jacq.
Recibido: 17 Julio 2019

Aceptado: 27 Noviembre 2019

Publicado: 25 Marzo 2020

Editor: Diego Gutierrez (iD

ISSN versión impresa 0373-580X ISSN versión on-line 1851-2372 
Sus miembros son morfológicamente diversos y ecológicamente importantes en comunidades de plantas de ambientes abiertos, áridos y semiáridos de regiones templadas de Sudamérica (Fernández et al., 2017).

En el contexto de la revisión de la subfamilia Azorelloideae para el proyecto de Flora Argentina, se encontraron 15 nombres pertenecientes a los géneros Asteriscium, Bolax, Bowlesia, Diposis, y Pozoa, que requieren tipificación. Aquí, resolvemos las tipificaciones de todos estos nombres.

\section{Materiales y Métodos}

Para realizar las tipificaciones, se examinaron los protólogos y el posible material original de todos los nombres. Se analizó material depositado en los herbarios de B, BM, CORD, E, F, G, GH, HAL, HBG, K, LAU, LP, MPU, NY, P, S, SGO, SI, UC y US (Thiers, 2019), disponible en la página de JSTOR (http://plants.jstor.org), a través de las bases de datos on-line de varios herbarios extranjeros, a través de imágenes digitales enviadas por los curadores de los herbarios o por la observación directa de los ejemplares. Todas las decisiones se basaron siguiendo el Código Internacional de Nomenclatura para Algas, Hongos y Plantas (ICN; Turland et al., 2018). Los ejemplares seleccionados como lectotipos o neotipos se indican por su código de barras. La información proporcionada para los tipos designados se refiere exactamente a la información de la etiqueta que se muestra en los ejemplares de herbario. Entre corchetes se detalla la información que no figura en las etiquetas y que proviene del análisis conjunto de etiquetas y protólogo, y entre comillas se detalla la información del protólogo cuando es necesario.

\section{Resultados}

Se designan 14 lectotipos y un neotipo, y se justifica la elección de los mismos. Los nombres tratados se colocan bajo los respectivos nombres actualmente aceptados que se presentan en orden alfabético (Martínez, 2008; Martínez \& Calviño, 2019). Se indican únicamente los tipos de los nombres que requieren tipificación.
1. Asteriscium argentinum Chodat \& Wilczek, Bull. Herb. Boissier, ser. 2, 2: 526. 1902. Tipo: [Argentina. Mendoza, Dpto. Malargüe], Collines arides sur l'Arroyo-Manga, entrée de la vallée de l'Atuel, c. 1800 m, Wilczek 63 (Lectotipo aquí designado G 00359789 [foto!], isolectotipo US 00126963 [foto!; fragmento]).

Los ejemplares tipo de Chodat principalmente están depositados en $\mathrm{G}$, y los de Wilczek principalmente en LAU aunque también en US, entre otros (Stafleu \& Cowan, 1976-1988). Mathias \& Constance (1962) indicaron haber visto material de Asteriscium argentinum en LAU; nosotras no localizamos dicho material pero sí encontramos ejemplares de esta especie depositados en G y US que son duplicados de la misma colección. Los dos ejemplares se ajustan correctamente al protólogo en cuanto a su morfología, al lugar de colección, al colector y número de colección, y presentan etiqueta original de los autores. Designamos como lectotipo de $A$. argentinum al ejemplar de $\mathrm{G}(\mathrm{G}$ 00359789) ya que se encuentra mejor conservado y presenta mayor cantidad de material vegetal que su duplicado en US, que además no presenta umbelas floríferas ni fructíferas.

2. Asteriscium fimbriatum Speg., Revista Fac. Agron. La Plata 3: 523. 1897. Tipo: [Argentina. Santa Cruz], "In pratis uliginosis dumosis prope Lago Argentino", 1884, Tonini del Furia s.n. (Holotipo LP, no localizado). Argentina. Neuquén, Dpto. Minas, Pasando Cancha Huinganco, $1500 \mathrm{~m}$ s.n.m., 25-I-2007, Urtubey \& Simon 318 (Neotipo aquí designado SI 045684!, isoneotipo SI 127464!).

Spegazzini (1897) describe a Asteriscium fimbriatum a partir de una única colección realizada por Tonini del Furia ("T.F.") en Lago Argentino (Santa Cruz, Argentina). Dado que no pudimos localizar este material ni en LP (com. pers. Liliana Katinas) ni en ningún otro herbario que tiene colecciones de Spegazzini (por ejemplo, BAB, SI), designamos un neotipo que servirá de tipo nomenclatural mientras el material original siga extraviado. Entre el material localizado, que coincide perfectamente con el protólogo en morfología, no encontramos colecciones en la provincia donde supuestamente fue coleccionado el material original. Designamos como neotipo al 


\section{Fernández y C. I. Calviño - Tipificaciones en Azorelloideae para la Flora de Argentina}

ejemplar SI 045684. El mismo fue coleccionado en Neuquén, se encuentra muy bien conservado $\mathrm{y}$ es un ejemplar completo con hojas basales y caulinares, y umbelas floríferas y fructíferas.

3. Bolax bovei (Speg.) Dusén, Rep. Princeton Univ. Exped. Patagonia, Botany 8, Suppl.: 195. 1914. Azorella bovei Speg., Anales Mus. Nac. Buenos Aires 5: 58. 1896. Tipo: [Chile. Magallanes y Antártica Chilena], Burnt Island, sin colector (Lectotipo aquí designado LP 002786 [foto!]).

En el protólogo de Azorella bovei, Spegazzini (1896) indica colecciones de esta especie en tres pequeñas islas del archipiélago de Tierra del Fuego: Isla Melville, Isla Basket e Isla Burnt. Estas islas son parte de la Región XII de Magallanes y Antártica Chilena, Chile. De estas colecciones, sólo localizamos un sintipo de la Isla Burnt depositado en LP y proveniente del herbario de Spegazzini. El ejemplar no presenta información del colector ni de la fecha de colección, pero se ajusta correctamente al protólogo en morfología y lugar de colección y lo designamos como lectotipo de Azorella bovei.

4. Bolax gummifera (Lam.) Spreng., Sp. Umbell.: 10. 1818. Hydrocotyle gummifera Lam., Encycl. 3: 156. 1789. Tipo: [Chile]. Estrecho de Magallanes, I-1768, Commerson s.n. (Lectotipo aquí designado P 00835001 [foto!], isolectotipos G 00367677 [foto!], MPU 019650 [foto!], MPU 019651 [foto!], MPU 019652 [foto!], MPU 019653 [foto!], MPU 019654 [foto!], P 00835002 [foto!], P 00835003 [foto!], P 00835004 [foto!], P 00835005 [foto!], SI 000737!).

= Bolax complicatus Spreng., Sp. Umbell.: 9. 1818. Tipo: [Chile]. "Terra magellanica", I-1769, Banks \& Solander s.n. (Lectotipo aquí designado US 00126958 [foto!; fragmento]).

El protólogo de Hydrocotyle gummifera indica que este taxón fue coleccionado por Commerson en las Islas Malvinas y en el Estrecho de Magallanes, por lo que la descripción de la nueva especie estaría basada en sintipos. Localizamos ejemplares del Estrecho de Magallanes depositados en G, MPU, $\mathrm{P}$ y SI (todos duplicados de la misma colección) que presentan etiquetas originales de Commerson y que concuerdan con el protólogo en morfología. Lamarck (1789) en el protólogo de Hydrocotyle gummifera indica que esta especie corresponde a lo que Commerson identificó en herbarios como "Bolax glebaria", un nombre no publicado. Entre el material original localizado, designamos a $\mathrm{P}$ 00835001 como lectotipo de H. gummifera. Este ejemplar también presenta una etiqueta original que indica "Bolax glebaria Commers." y una etiqueta con una descripción morfológica original que concuerda con el protólogo.

El protólogo de Bolax complicatus (Sprengel, 1818) no cita ningún ejemplar o herbario en particular, pero hace el siguiente comentario "Chamitis complicata Banks in herbar. Forster. Azorella Lam. ill. t. I89. f.2.? Habitat in terra magellanica, unde Forsterus attulit. (v. s.)". Gran parte del material de Forster padre e hijo, fue adquirido por Banks y luego conservado en BM (Brownsey, 2012). No localizamos material original en BM (com. pers. Ranee Prakash), pero sí un ejemplar en el herbario US (US 00126958), que es un fragmento del material original que estuvo depositado en BM. El mismo, presenta etiquetas que indican "Ex Herbario Musei Britannici," "fragment material in closed pocket," y "Chamitis complicata Ms.". Designamos al ejemplar de US como lectotipo de Bolax complicatus.

5. Bowlesia incana Ruiz \& Pav., Fl. Peruv. Chil. 3: 28, tab. 268, fig. a. 1802.

= Bowlesia tenera Spreng., Syst. Veg. 1: 880 . 1825. Bowlesia incana Ruiz \& Pav. f. tenera (Spreng.) Urb., Fl. Bras. 11(1): 292. 1879. Tipo: [Uruguay]. Montevideo, Sellow s.n. (Lectotipo aquí designado BM 001008514 [foto!], isolectotipo F 0047978F [foto!]).

= Bowlesia nodiflora DC., Prodr. 4: 75. 1830. Tipo: Chile. Localidad no indicada, Poeppig 94 [Diar. 248] (Lectotipo aquí designado G 00661015 [foto!], isolectotipos BM 001008520 [foto!], F 0047974F [foto!], GH 00075425 [foto!], HAL 0026859 [foto!]).

El protólogo de Bowlesia tenera (Sprengel, 1825) menciona una colección realizada por Sellow en Montevideo. Localizamos ejemplares en BM, F, GH y L que coinciden con el protólogo en morfología y colector. Sin embargo, los ejemplares de GH y de L no pueden ser considerados con certeza duplicados de los especímenes de BM y F ya que no coincide el lugar de colección: BM 001008514 y F 0047978F 
indican "Montevideo" mientras que GH 00075428 y L 0008346, "Brasilia". Ambos ejemplares de Montevideo presentan etiquetas originales; entre éstos designamos a BM 001008514 como lectotipo de $B$. tenera por ser un ejemplar muy bien conservado que presenta abundante material y varias umbelas fructíferas.

El protólogo de Bowlesia nodiflora (De Candolle, 1830) indica que el nombre fue usado por Presl en ejemplares colectados por Haenke, y cita dos colecciones: "in montanis Peruviae Huanaccensis legit. cl. Haenke et in Chili cl. Poeppig, qui pro B. lobata habet", por lo que se infiere que hay sintipos, y por lo tanto es necesario lectotipificar. Analizamos ejemplares de ambas colecciones: Haenke (G 00661021) y Poeppig (BM 001008520, F 0047974F, G 00661015, GH 00075425 y HAL 0026859) que coinciden perfectamente con el protólogo en morfología y que presentan etiquetas que fueron vistas por Presl y determinadas por él como B. nodiflora. Designamos a G 00661015 como lectotipo de $B$. nodiflora ya que proviene del herbario de De Candolle y presenta abundante material con varias umbelas fructíferas.

6. Bowlesia lobata Ruiz \& Pav., Fl. Peruv. Chil. 3: 28, pl. 251, fig. b. 1802.

= Bowlesia acutiloba H. Wolff., Bot. Jahrb. Syst. 40: 284. 1908. Tipo: Bolivia. Tarija, Calderillo, 3400 m, 23-III-1904, Fiebrig 3168 (Lectotipo aquí designado G 00367592 [foto!], isolectotipos BM 001008526 [foto!], F 0047968F [foto!; fragmento], K 000529707 [foto!]).

En el protólogo de Bowlesia acutiloba, Wolff (1908) menciona una única colección, Fiebrig 3168, de Bolivia. Localizamos varios duplicados del material original. Ninguno de estos duplicados presenta etiquetas de identificación o evidencia explícita por parte de Wolff; es posible que el holotipo haya estado en $\mathrm{B}$, herbario donde trabajaba el autor, aunque allí no fue localizado (com. pers. con Robert Vogt). Designamos a G 00367592 como lectotipo de $B$. acutiloba ya que es un ejemplar completo y con abundante material vegetativo y reproductivo.

7. Bowlesia tropaeolifolia Gillies \& Hook., Bot. Misc. 1: 325. 1830. Tipo: [Argentina]. Mendoza, "in umbrosis et in fissuris rupium apud "el Salto de San
Isidro," in convalle Andium versus Mendozam: alt. 6000 ped.", Gillies s.n. (Lectotipo aquí designado $\mathrm{K} 000529702$ [foto!], isolectotipos E 00000029 [foto!], E 00000030 [foto!], E 00000031 [foto!]).

= Bowlesia pulchella Wedd., Chlor. Andina 2: 188, pl. 67, B. 1860. Tipo: Chile-Perú. Arica y Parinacota-Tacna, Cordillera de Tacora, 1851, Weddell s.n. (Lectotipo aquí designado P 01817061 [foto!], isolectotipo F 0047977F [foto!; fragmento]).

= Bowlesia cirrosa Phil., Anales Univ. Chile 85:

515. 1894. Bowlesia tropaeolifolia Gillies \& Hook. var. cirrosa (Phil.) Reiche, Anales Univ. Chile 104: 783. 1899. Tipo: Chile. Nubble, Chillán, Cueva de Pincheira, I-1887, Philippi s.n. (Lectotipo aquí designado SGO 000003675 [foto!], isolectotipo SGO 000003676 [foto!]).

= Bowlesia tropaeolifolia Gillies \& Hook. var. patagonica Speg., Anales Mus. Nac. Buenos Aires 7: 294. 1902. Tipo: Argentina. Chubut, Corcovado, Nafofo-Cahuellu, 10/12-III-1901, Illin 249 (Lectotipo aquí designado LP 080897 [foto!], isolectotipos CORD 00003534!, HBG 517907 [foto!], SI 131881!).

$=$ Bowlesia tropaeolifolia Gillies \& Hook. var. gayana Domin, Repert. Spec. Nov. Regni Veg. 4: 299. 1907. Tipo: Chile. Localidad no indicada, Gay 828 (Lectotipo aquí designado F 0047979F [foto!], isolectotipos G 00367597 [foto!], NY 00405741 [foto!]).

Encontramos cuatro ejemplares de Bowlesia tropaeolifolia que coinciden perfectamente con el protólogo y todos presentan etiquetas originales de Gillies. De éstos, el ejemplar de K (K 000529702) tiene un sello del "Herbarium Hookerianum", y dos de los ejemplares de E (E 00000029 y E 00000030) tienen etiquetas con el nombre del taxón seguido por "nov. spec." con letra de Gillies. Designamos a K 000529702 como lectotipo de B. tropaeolifolia ya que está muy bien conservado y presenta abundante material vegetal.

En el protólogo de Bowlesia pulchella, Weddell (1860) indica dos colecciones: una en Perú realizada por Weddell, "creux des rochers, dans les parties les plus élevées des Andes du département de Tacna (Wedd.)" y otra en Bolivia por d'Orbigny "fentes des rochers, au niveau des neiges de la Quebrada de las Lagunas de Potosí (d'Orbigny)". Encontramos material original de Perú coleccionado por Weddell (F 0047977F y P 01817061). Ambos 


\section{Fernández y C. I. Calviño - Tipificaciones en Azorelloideae para la Flora de Argentina}

ejemplares provienen originalmente del herbario $\mathrm{P}$ donde trabajaba el autor de la especie; el ejemplar de $\mathrm{F}$ presenta etiquetas que indican "Ex Herbario Musei Parisiensis" y "frag. type", por lo que es un fragmento del ejemplar depositado en P. Designamos a P 01817061 como lectotipo de B. pulchella ya que está bien preservado y presenta abundante material.

Encontramos dos ejemplares en SGO que coinciden con el protólogo de Bowlesia cirrosa en morfología, localidad y colector, y ambos presentan etiquetas originales escritas por Philippi, por lo que constituyen material original utilizado por el autor para describir la nueva especie. Designamos a SGO 000003675 como lectotipo de B. cirrosa; presenta más material que el otro duplicado y varias umbelas fructíferas.

El protólogo de Bowlesia tropaeolifolia var. patagonica indica las siguientes dos colecciones: “in pratis secus Carren-leofú, aest. 1899” y "prope Nafofo-cahuellu, Chubut, aest. 1901 (N. I.)", por lo que hay sintipos y es necesario lectotipificar. Analizamos material original de ambas colecciones: Carren-leofú (LP 80895) y de Nafofo-Cahuellu (CORD 00003534, HBG 517907, LP 080897 y SI 131881). Todos los ejemplares coinciden con el protólogo en morfología, colector, localidad y fecha de colección. De estos ejemplares, designamos a LP 080897 como lectotipo de B. tropaeolifolia var. patagonica porque pertenecía al herbario de Spegazzini (ex LPS 24093) y porque presenta etiquetas originales del colector.

El protólogo de Bowlesia tropaeolifolia var. gayana indica que este taxón fue coleccionado por Gay en Chile. Encontramos varios ejemplares coleccionados por Gay en ese país que coinciden también con el protólogo en morfología y lugar de colección, por lo que los consideramos material original (Gay 828 F 0047979F, G 00367597, NY 00405741; Gay s.n. GH 00075429, NY 00405742 , NY 00405743). Entre éstos, designamos como lectotipo de B. tropaeolifolia var. gayana a F 0047979F; ya que es un ejemplar completo con abundante material. Este ejemplar, junto con $\mathrm{G}$ 00367597 y NY 00405741 son duplicados de una misma colección (Gay 828), mientras que los ejemplares GH 00075429 , NY 00405742 y NY 00405743 no tienen número de colección, y por lo tanto podrían provenir de colecciones diferentes por lo que no los consideramos duplicados del lectotipo seleccionado.
8. Diposis patagonica Skottsb., Kungl. Svenska Vetenskapsakad. Handl. 56: 278. 1916. Tipo: Argentina. Chubut, Patagonia andina, Cerro Lelej, 800 m, 29-X-1908, Skottsberg 542 (Lectotipo aquí designado S 05-4071 [foto!], isolectotipo SGO 000003691 [foto!]).

En el protólogo de Diposis patagonica, Skottsberg cita dos colecciones diferentes: "Subandines Patagonien: Terr. Chubut, Cerro Lelej, c. $800 \mathrm{~m}$, in der Steppe (Bl. 2, 29. 10. 08)" y "s. von Estancia Lelej (Bl. 2, 3. 11. 08)". Encontramos material original del Cerro Lelej (S 05-4071 y SGO 000003691). Ambos ejemplares coinciden con el protólogo en morfología y localidad, y presentan una etiqueta con el nombre de la especie seguido por "nov. sp. Skottsberg", escrito posiblemente por el propio autor. Seleccionamos como lectotipo de $D$. patagonica al ejemplar S 05-4071 que está completo y mejor preservado que su duplicado.

\section{Pozoa coriacea Lag., Gen. Sp. Pl. 13. 1816.}

= Pozoa hydrocotylifolia Bridges ex Fielding \& Gardner, Sert. Pl.: 170, tab. 40. 1844. Azorella hydrocotylifolia (Fielding \& Gardner) Macloskie, Rep. Princeton Univ. Exped. Patagonia, Botany 8: 629. 1905. Tipo: [Argentina. Mendoza], "In the bed of mountain rivers, on the eastern side of the Chilean Andes", Valle de las Cuevas, Bridges 1190 (Lectotipo aquí designado K 000529660 [foto!], isolectotipos BM 001008504 [foto!], E 00070644 [foto!], K 000529659 [foto!]).

El protólogo de Pozoa hydrocotylifolia cita la siguiente colección: "In the bed of mountain rivers, on the eastern side of the Chilian Andes. Bridges, n. 1190". Encontramos varios ejemplares que coinciden perfectamente con el protólogo en morfología, colector y número de colección (BM 001008504, E 00070644, K 000529659, K 000529660, P 00115460). Sin embargo, uno de estos ejemplares (P 00115460) tiene una etiqueta con fecha de colección posterior a la de la publicación de la nueva especie (1866) y dice Coquimbo, por lo tanto no puede considerarse parte del material original. Entre los restantes, designamos como lectotipo a K 000529660 ya que este duplicado, coleccionado en Valle de las Cuevas, una localidad mendocina al este de los Andes chilenos, se encuentra mejor preservado. 


\section{Contribución DE LOS AUTORES}

Las autoras realizaron conjuntamente y en partes iguales la colecta de datos, su interpretación y la redacción del manuscrito.

\section{Agradecimientos}

Agradecemos a Susana Martínez por valiosas discusiones sobre conflictos de tipificación. A los curadores de los herbarios B, BM, G, LP y US por facilitar la consulta y/o el envío de fotografías de los ejemplares estudiados aquí. A Diego G. Gutiérrez y a dos revisores anónimos por sus comentarios y sugerencias para mejorar el manuscrito. Este trabajo fue financiado por CONICET PIP 112201301-00357, y por la Universidad Nacional del Comahue PIN B205.

\section{Bibliografía}

BROWNSEY, P. J. 2012. The Banks and Solander collections-a benchmark for understanding the New Zealand flora. J. Roy. Soc. New Zealand 42: 131-137.

DE CANDOLLE, A. P. 1830. Prodromus systematis naturalis regni vegetabilis 4 . Treuttel et Würtz, Paris.

FERNÁNDEZ, M., C. EZCURRA \& C. I. CALVIÑO. 2017. Chloroplast and ITS phylogenies to understand the evolutionary history of the southern South American Azorella, Laretia and Mulinum (Azorelloideae, Apiaceae). Mol. Phylogenet. Evol. 108: 1-21.

LAMARCK, J. B. 1789. Encyclopédie Méthodique. Botanique. Tome Troisieme. Chez Panckoucke, Paris.

MARTÍNEZ, S. 2008. Apiaceae. En: ZULOAGA, F. O., O. MORRONE \& M. J. BELGRANO (eds.), Catálogo de las Plantas Vasculares del Cono Sur (Argentina, sur de Brasil, Chile, Paraguay y Uruguay), vol. 2, pp. 1056-1090. Monographs in Systematic Botany from the Missouri Botanical Garden, St. Louis.

MARTÍNEZ, S. \& C. I. CALVIÑO. 2019. Apiaceae. En: ZULOAGA, F. O. \& M. J. BELGRANO (eds.),
Flora vascular de la República Argentina, vol. 20, pp. 1-122. IBODA, CONICET, San Isidro.

MATHIAS, M. \& L. CONSTANCE. 1962. A revision of Asteriscium and some related hydrocotyloid Umbelliferae. Univ. Calif. Publ. Bot. 33: 99-184.

NICOLAS, A. N. \& G. M. PLUNKETT. 2009. The demise of subfamily Hydrocotyloideae (Apiaceae) and the re-alignment of its genera across the entire order Apiales. Mol. Phylogenet. Evol. 53: 134-151.

PLUNKETT, G. M. \& A. N. NICOLAS. 2017. Assessing Azorella (Apiaceae) and its allies: phylogenetics and a new classification. Brittonia 69: 31-61.

SPEGAZZINI, C. L. 1896. Plantae per Fuegiam anno 1882 collectae. Anales Mus. Nac. Buenos Aires 5: 39-104.

SPEGAZZINI, C. L. 1897. Plantae Patagoniae Australis. Revista Fac. Agron. La Plata 3: 485-589.

SPRENGEL, C. 1818. Species Umbelliferarum minus cognitae. Halae in officina Rengeriana, Göttingen.

SPRENGEL, C. 1825. Systema Vegetabilium 1. Sumtibus Librariae Dieterichianae, Göttingen.

STAFLEU, F. A. \& R. S. COWAN. 1976-1988. Taxonomic Literature II: A selective guide to botanical publications and collections with dates, commentaries and types. Disponible en https:// www.sil.si.edu/DigitalCollections/tl-2/search.cfm [Acceso: 4 Septiembre 2019].

THIERS, B. 2019. Index Herbariorum: A global directory of public herbaria and associated staff. New York Botanical Garden's Virtual Herbarium. Disponible en http://sweetgum.nybg.org/ih/: [Acceso: 26 Julio 2019].

TURLAND, N. J., J. H. WIERSEMA, F. R. BARRIE, W. GREUTER, D.L. HAWKSWORTH, P. S. HERENDEEN, S. KNAPP, W.-H. KUSBER, D.Z. LI, K. MARHOLD, T. W. MAY, J. MCNEILL, A. M. MONRO, J. PRADO, M. J. PRICE \& G. F. SMITH. 2018. International Code of Nomenclature for algae, fungi, and plants (Shenzhen Code), Regnum Veg. 159. Koeltz Botanical Books, Glashütten.

WEDDELL, H. A. 1860. Chloris Andina. Essai d'une flore de la région alpine des Cordillères de l'Amérique du Sud. Vol. 2. Chez P. Bertrand, Paris.

WOLFF, H. 1908. Umbelliferae austro-americanae. Bot. Jahrb. Syst. 40: 281-306. 